\title{
IN VITRO SYNTHESIS OF PARTICULATE GLYCOGEN FROM URIDINE DIPHOSPHATE GLUCOSE ${ }^{1}$
}

\author{
A. J. Parodi, J. Mordoh, ${ }^{2}$ Clara R. Krisman and L. F. Leinlo \\ Instituto de Investigaciones Bioquimicas, Fundación Campomar \\ and Facultad de Ciencias Exactas y Naturales, Obligado 2490, Buenos Aires, Argantina
}

\begin{abstract}
High molecular weight glycogen has been prepared in vitro with liver glycogen synthetase (uridine diphosphate glucose: $\alpha$-1,4glucan $\alpha$-4-glucosyltransferase, FC 2.4.1.11) and branching enzyme ( $\alpha$-1,4-glucan: $\alpha$-1,4 glucan 6-glycosyltransferase, EC 2.4.1.18) and uridine diphosphate glucose as glucose donor. The product obtained did not differ significantly from the native glycogen as judged by iodine spectrum, sedimentation coefficient in sucrose gradients, and by the effect of treatment with acid or alkali. Glycogen obtained from uridine diphosphate glucose differed from that prepared with glucose 1-phosphate as glucosyl donor.
\end{abstract}

The molecular weight of liver glycogen extracted in the cold and at neutral $\mathrm{pH}$ is very high $(1-5)$. Values of 10-1000 million daltons have been reported $(4,5)$. Samples of the same molecular weight have been obtained in vitro by incubating glc-l.P3, crystalline phosphorylase (a-1,4-glucan: orthophosphate glucosyltransferase, EC 2.4.1.1.) and purified liver branching enzyme $(\alpha-1,4$ glucan 6 glycosyltransferase, EC 2.4.1.18) (6) but further work showed that this glycogen is different from that isolated from liver $(5,7)$. Glycogen preparerl with phosphorylase (glc-1-P-glycogen ${ }^{4}$ ) was found to be more stable to heat, acid and alkali. The degradation of native glycogen under such conditions gave 'rise preferentially to molecules of about 8 million daltons whereas glc-1-P. glycogen was degraded with a progressive decrease in molecular weight.

\section{Observations with the electron microscope}

1 This investigation was supported in part by a research grant (No. GM 03442) from the National Institutes of Health, U. S. Public Health Service, by The Rockefeller Foundation and by The Consejo Nacional de Investigaciones Científicas y Técnicas (R. Argentina).

2 Career investigator of the Consejo Nacional de Investigaciones Científicas y Técnicas.

3 The following abbreviations are used: glc-1-P, dglucose 1-phosphate; UDPG, uridine diphosphate glu. cose,

4 For simplicity glycogens synthesized in vitro from glc-1-P and phosphorylase or from UDPG and glycogen synthetase will be referred to as glc-1-P-glycogen and UDPG-glycogen, respertivel. showed that native glycogen appeared to have more loosely joined subparticles, giving a more open structure (7).

This paper reports the preparation and properties of glycogen obtained using UDPG and glycogen synthetase (UDPG: $\alpha$-1,4-glucan a-4-glucosyltransferase, EC 2.4.1.11). The samples obtained in this manner were very similar to those of native liver glycogen as judged by the rate of sedimentation and by the effect of acid or alkali.

\section{MATERIALS AND METHODS}

Materials. The synthesis of glc-1-P and UDPG was carried out according to MacDonald (8) and Moffiat (9) respectively. Yeast UDPG was a gift of C. F. Boehringed \& Soehne (Germany) or was purchased from Sigma Chemical Co. (USA). Radioactive UDPG was obtained from the Radiochemical Centre (England); bovine pancreatic ribonuclease $5 \times$ crystalline type I-A was purchased from Sigma Chemical Co. (USA) and was dissolved (10 $\mathrm{mg}$ per $\mathrm{ml}$ ) in glycylglycine buffer of $\mathrm{pH}$ 7.75. Native liver glycogen was obtained by extraction with phenol as described by Laskov and Margoliash (10), with some modifications. The preparation of glycogen from glc-1-P was carried out as described by Mordoh et al. (6).

Analytical. Molecular weight determinations were carried out by centrifugation in sucrose gradients as previously described (5). The caliuration data were the same. Radioactivity was measured using Bray (11) solution and a scintillation colnter. Glycogen was measured according to Krisman (12). Glycogen synthetase was assayed as described by Rothman and Cabib (13) with some modifications.

Enzymes. Rat-liver branching enzyme was prepared as described by Krisman (14) but the livers were thoroughly perfused with $250 \mathrm{~mm}$ sucrose, $5 \mathrm{~mm}$ 
EDTA. Crystalline phosphorylase $b$ from rabbit muscle was obtained according to Fischer et al. (15).

Liver glycogen synthetase. This enzyme was prepared as follows: Rats weighing about $300 \mathrm{~g}$ were fasted for $48 \mathrm{hr}$. In order to decrease glycogen content as much as possible, insulin, which is known to produce glycogenolysis in liver, was injected intraperitoneally (20 units) and the animals were killed 1 hr later. The excised livers were perfused with a solution containing $250 \mathrm{~mm}$ sucrose, $10 \mathrm{~mm}$ mercaptoethanol, $5 \mathrm{~mm}$ EDTA, and then homogenized in 5 vol of a similar solution but containing $880 \mathrm{~mm}$ sucrose. The homogenate was centrifuged at $96,000 \mathrm{~g}$ for $3 \mathrm{hr}$. The precipitate was discarded and the supernatant fluid was centrifuged at $150,000 \mathrm{~g}$ for $3 \mathrm{hr}$. The small pellet was resuspended (three livers in about $1 \mathrm{ml}$ ) in the solution used for homogenization. The resulting preparation was found to be stable for weeks when kept at $-200^{\circ}$ and could be frozen and thawed repeatedly without loss of glycogen synthetase activity. It had some branching activity and only traces of $\alpha$-amylase. No glycogen was detectuble in the preparations as judged by colorimetric tests but acceptor activity could be detected by carrying out measurements of glycogen synthetase lactivity without added glycogen. The molecular weight of this endogenous acceptor must be less than 5 million daltons since glycogen of that molecular weight is $90 \%$ sedimentable when centrifuged at $96,000 \mathrm{~g}$ for $3 \mathrm{hr}$ in $880 \mathrm{~mm}$ sucrose.

Glycogen synthesis from UDPG. A typical incubation mixture contained $0.11 \mathrm{M}$ glycylglycine buffer of pIf 7.75, $44 \mathrm{~mm}$ mercaptoethianol, $5.5 \mathrm{~mm}$ glucose 6 phosphate, $22 \mathrm{~mm}$ EDTA ( $\mathrm{pH} 7.0$ ), $0.02 \mathrm{mg}$ of KOHglycogen, 0.1 M UDPG, $10 \mu \mathrm{l}$ of ribonuclease solution, $150 \mu 1$ of glycogen synthetase and $150 \mu$ l of the branching enzyme, in a total volume of $450 \mu$ l. The presence of chloride ions was avoided in order to keep $\alpha$-amylase activity as low as possible.

After $5 \mathrm{hr}$ lat $37^{\circ}$, water was added to make $5 \mathrm{ml}$ and the mixture was centrifuged at $2,000 \mathrm{rpm}$ for $5 \mathrm{~min}$. The supernatant fluid was shaken with $2 \mathrm{ml}$ of water-saturated phenol and centrifuged as before; the phenol was re-extracted with $5 \mathrm{ml}$ of water. The two aqueous layers were mixed and 2 vol of ethanol were added. The precipitate was dissolved in water and dialyzed overnight against water in the cold room. The supernatant fluid obtained after centrifugation $(2,000 \mathrm{rpm}, 10 \mathrm{~min})$ was precipitated with ethanol. A drop of saturated ammonium acetate was added in order to obtain good precipitation.

\section{RESULTS}

Properties of glycogen prepared from UD. $P G$. Previous attempts to prepare high molecular weight glycogen with UDPG and glycogen synthetase failed because the enzyme preparations were not active enough or contained amylase. Under the conditions given in $\mathrm{Me}$ thods glycogen is formed with a yield which often reached $50 \%$ of the theoretical based on UDPG added. The glycogen formed was difficult to free from nucleic acids and, to facilitate purification, ribonuclease was added to the reaction mixture.
The iodine spectrum of the product formed was almost the same as that of native gly. cogen and clearly different from that of amylopectin (Fig. 1), Treatment with salivary $\alpha$-amylase gave rise, as expected, to the formation of maltotriose and maltose and under the action of $\beta$-amylase, maltose was formed.

The molecular weight distribution of a sample is shown in Fig. 2. Most of the substance is of very high molecular weight. The curves varied in different experiments (see also Fig. 4A) but did not differ much from those of native liver glycogen.

A comparison of native, UDPG-glycogen and glc-1-P-glycogen was carried out by measuring the decrease in absorbance in $0.1 \mathrm{~N}$ acid at room temperature or in $0.1 \mathrm{~N}$ alkali at 100 . Previous work $(5,7)$ had shown that under these conditions glc-1-P-glycogen can be clearly distinguished from native samples, because the latter is more labile. As shown in Fig. 3, UDPG-glycogen and native glycogen were found to behave exactly the same, whereas the turbidity of glc-1-P-glycogen decreased more slowly.

Another way of following the degradation of glycogen is by gradient centrifugation. Previous work $(5,7)$ had shown that acid or alkali treatment of native glycogen gives rise preferentially to molecules of about 8 million daltons, whereas glc-P-glycogen showed a progressive decrease in molecular weight.

The action of acid on native glycogen and ElDPG-glycogen is shown in Fig. 4. Before

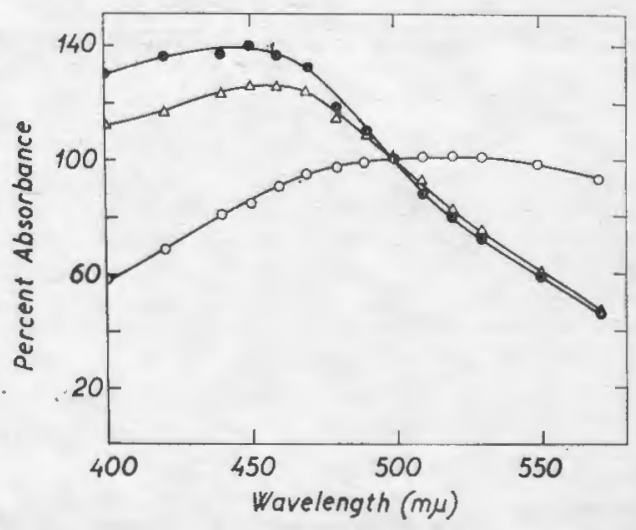

FIG. 1. - Iodine spectra of liver glycogen, amylopectin and UDPG-glycogen. Conditions as described by Krisman (12). Absorbances at $500 \mathrm{~m} \mu$ are taken as $100 \%$. Full circles: native liver glycogen; empty circles: amylopectin; triangles: UDPG-glycogen. 


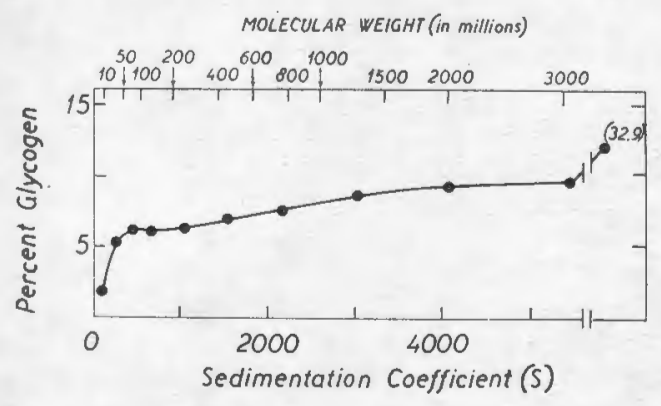

s'1G, 2. - Molecular weight distribution of glycogen prepared with UDPG as glucosyl donor as described in Methods. The number in parentheses represents the percentage glycogen in the pellet. This corresponds to glycogen of sedimentation coefficient higher than $5500 \mathrm{~S}$ plus that which sediments along the walls of the tube.

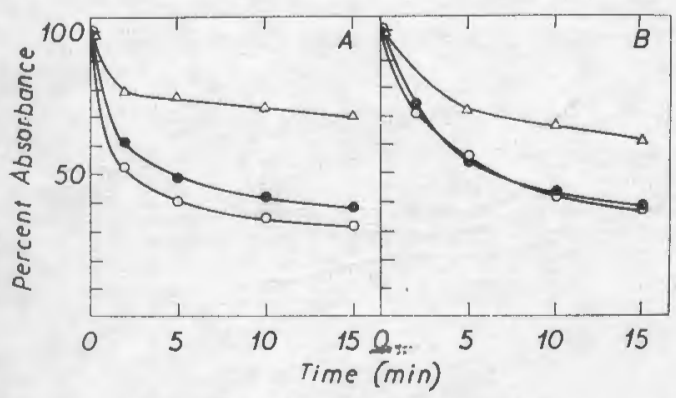

FIG. 3. - The action of acid and alkali on native glycogen, UDPG-glycogen and glc 1-P-glycogen. Absorbance was measured at $500 \mathrm{~m} \mu$. A: $0.1 \mathrm{~N}$ HCL at 25; B: $0.1 \mathrm{~N} \mathrm{NaOH}$ at 100 . Full circles: native glycogen; empty circles: UDPG-glycogen; triangles: glc1-P glycogen.

treatment both samples gave similar curves (Fig. 4A). After $30 \mathrm{sec}$ in $0.1 \mathrm{~N}$ acid at $37 \%$ a peak appeared at about $260 \mathrm{~S}$ (Fig. 4B) and after 5 min nearly all the population had become light (Fig. 4C). A fine comparison of the light peak was obtained by centrifuging for a longer time. In this way slight differences were detectable between the two samples. It can be observed in Fig. $4 \mathrm{D}$ that at $30 \mathrm{sec}$ the light peak of UDPG-glycogen is more polydisperse than that of the native. After a 5or $10-\mathrm{min}$ treatment (see Figs. $4 \mathrm{E}$ and $\mathrm{F}$ ) the peak values were $150 \mathrm{~S}$ and $100 \mathrm{~S}$ for UDPGglycogen and native glycogen, respectively. These values correspond to 13 and 8 million daltons. The results of alkaline treatment are shown in Fig. $5 \mathrm{~A}$ to D. Both native glycogen and UDPG-glycogen were appreciably degraded after $1 \mathrm{~min}$ and became progressively lighter. No appreciable difference between the two samples was observable. However, after $5 \mathrm{~min}$ in $0.1 \mathrm{~N}$ alkali at 100 the native sample showed a small peak at about 110 s which was absent in the sample synthesized in vitro. The result of a more drastic alkaline treatment $\left(33 \% \mathrm{KOH}\right.$ at $100^{\circ}$ for $\left.20 \mathrm{~min}\right)$ is shown in Fig. 6. Both samples gave rise to the formation of a glycogen of approximately the same molecular weight (8 million daltons).

A comparison of the decomposition of UD. PG- and glc-1-P-glycogens produced by heating at a pH of about 5 is shown in Fig. 7. It can be seen that the initial curves were very similar but after heating a peak at about $180 S$ was formed from UDPG-glycogen whereas glc-1-P-glycogen was only slightly degraded.

Treatment with $8 \mathrm{~m}$ urea, which is currently used for breaking hydrogen bonds, produced no appreciable changes on the UDPG-glycogen samples. The treatment was carried out at $37^{\circ}$ for $10 \mathrm{~min}$. It had been observed before (5) that native glycogen is slightly degraded after 7 days in $8 \mathrm{~m}$ urea.
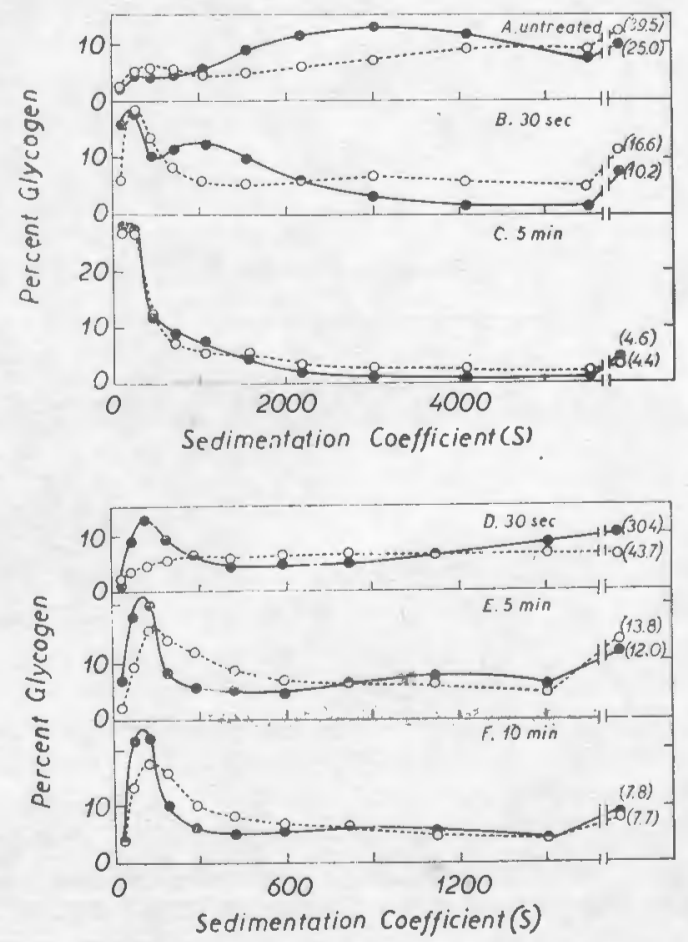

Fig. 4. - The action of acid on native glycogen and UDPG-glycogen. Full line: native glycogen; dotted line: UDPG-glycogen. A: before treatment; B-F: after indicated times in $0.1 \mathrm{~N} \mathrm{HCl}$ at $37 \%$. The centrifugation time was: A-C: $33 \mathrm{~min} ; \mathrm{D}-\mathrm{F}$ : $121 \mathrm{~min}$. 


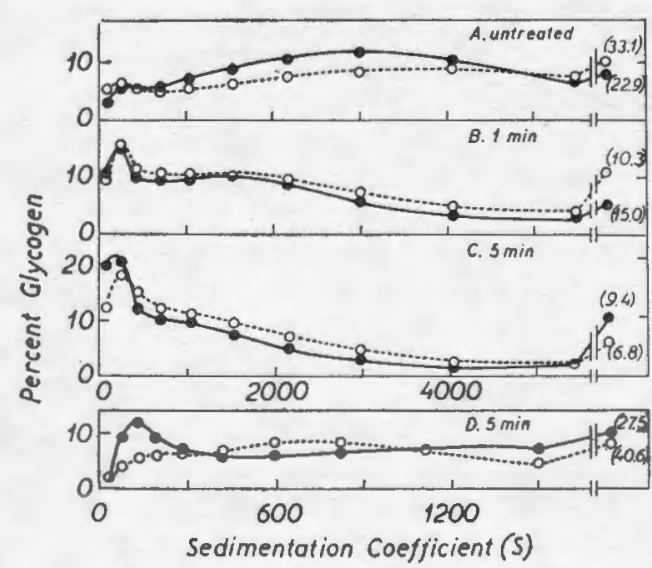

FIG. 5. - The action of alkali on native glycogen and UDPG-glycogen. Full line: native glycogen; dotted line: UDPG-glycogen. A: before treatment; B-D: after indicated times in $0.1 \mathrm{~N} \mathrm{NaOH}$ at 100 \%. The centrifugation time was: A-C: $33 \mathrm{~min}$; D: $121 \mathrm{~min}$.

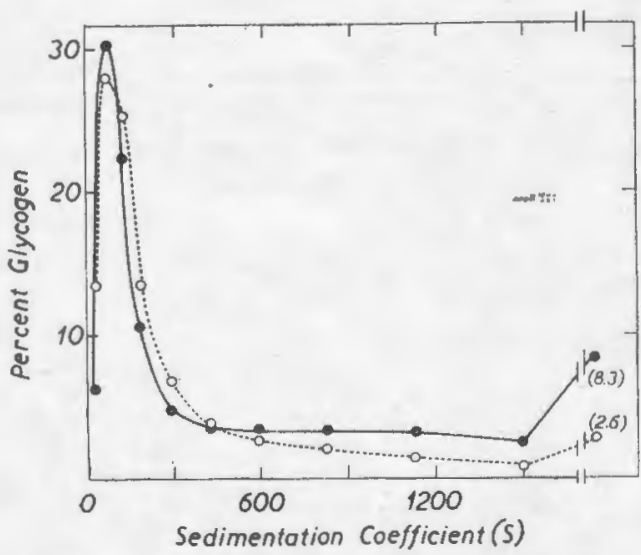

Fig. 6. - The action of alkali on native glycogen and UDPQ-glycogen. Full line: native glycogen; dotted line: UDPG-glycogen. The original samples were the same as those shown in Fig. 5A. They were treated with $33 \% \mathrm{KOH}$ for $20 \mathrm{~min}$ at 1000 . The analytical run in sucrose gradient was for $121 \mathrm{~min}$.

Effect of dilution. If high molecular weight glycogen is formed by entanglement of molecules during synthesis it might be reasoned that if the reaction is carried out in a dilute solution, the resulting glycogen should be lighter. The result 'of such an experiment is shown in Fig. 8. One of the incubation mixtures was diluted six-fold with water and incubated a longer time so as to reach the same final yield of glycogen. The diluted sample gave high molecular weight glycogen whereas the control did not become as heavy in the time allowed for the synthesis to take place. The results were, therefore, the opposite from those expected from the entanglement hypothesis. No explanation for the result has been yet found.

Glucose 1-phosphate as donor. The products obtained from glc-1-P and UDPG under the same conditions were studied. One sample was synthesized with UDPG as donor and another with exactly the same incubation mixture except that UDPG was replaced by glc-1-P plus phosphorylase and adenosine 5'-

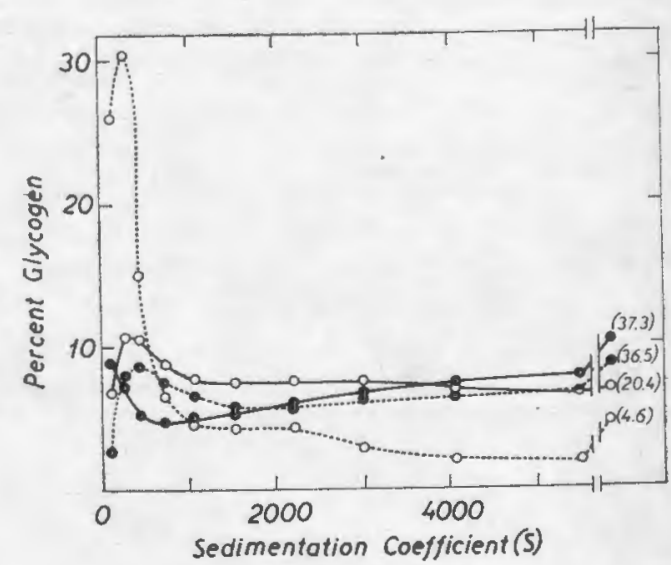

FIG. 7. - Lability of UDPG-and glc-1-P-glycogens. The samples were heated at 1009 for $15 \mathrm{~min}$ in $0.1 \mathrm{~m}$ Tris-HCl buffer of $\mathrm{pH} 7.2$ (the $\mathrm{pH}$ at $100^{\circ}$ is about 5). Full circles: before treatment; empty circles: after heating. Full line: glc-1-P_glycogen; dotted line: UDPG-glycogen.

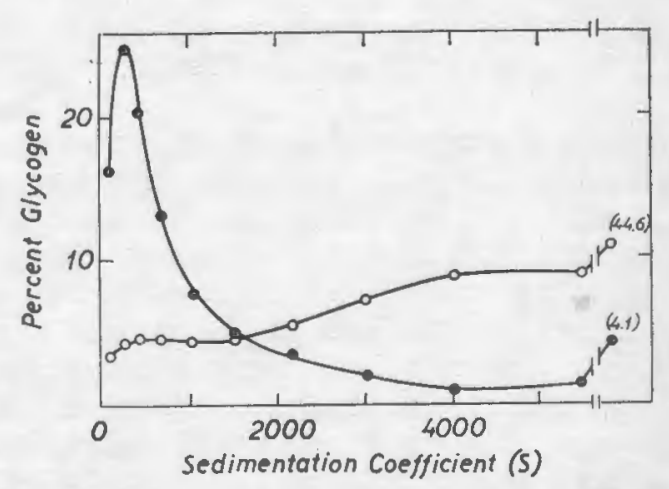

FIG. 8. - Glycogen synthesis in a diluted reaction mixture. Full circles: glycogen formed from UDPG as described in text. Empty circles: glyoogen formed with the same mixture but diluted six-fold with water. The incubation times were 30 and $96 \mathrm{~min}$, respectively. Radioactive UDPG was used and glycogen content was estimated by its radioactivity. Total synthesis was $15 \%$ higher in he unäiluted sample. 
monophosphate. Although the extent of synthesis was the same, the molecular weight distribution was completely different, the glc1-P-glycogen being lighter (Fig. 9). It was checked photometrically and by gradient centrifugation that the effect of acid on this sample was the same as on slc-1-P-glycogen prepared as previously described $(5,6)$. It should be pointed out that the glc-1-P-glycogen can reach higher sizes if $\mathrm{KOH}$-glycogen is omitted in the incubation mixture.

It can be predicted that the final molecular weight should be inversely proportional to the amount of acceptor molecules present when the reaction is started. Therefore one explanation of the fact that the glycogen formed with glc-1-P is lighter than that obtained from UDPG, would be that some reagents (glc-1-P, phosphorylase or adenosine 5 '-monophosphat€) contained acceptor molecules. This possibility was excluded by control experiments in which each of these substances were found to produce no changes in the molecular weight distribution when added to incubation mixture in which UDPG was the glucose donor.

Variations in the incubation mixture. The omission of glucose 6-phosphate in the incubation mixture did not:-change appreciably the molecular weight distribution. However since the glycogen synthetase is only about $80 \%$ active without glucose 6-phosphate the rate of synthesis was slightly slower. Ribonuclease was included in the incubation mixture

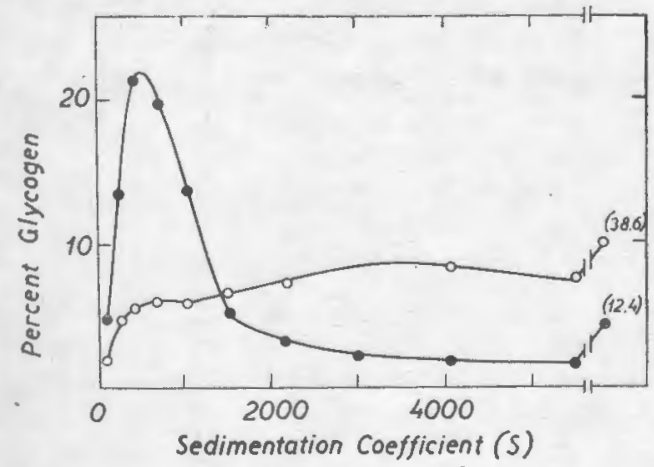

FIG. 9. - Molecular weight distributions of glycogen formed from glc-1-P and UDPG. Full circles: glc-1-P as glucose donor; empty circles: UDPG as glucose donor. The reaction mixtures were as described in Methods for UDPG but one sample contained synthetic glc-1-P (270 mM), adenosine 5'-monophosphate $(4.4 \mathrm{~mm})$ and crystalline phosphorylase $b$ instead of UDPG. The amount of glycogen formed was practically the same in boih cases. because difficulties were experienced in the purification of glycogen and the main contaminant appeared to be nucleic acids. The distribution curve of glycogen obtained with "or without ribonuclease was the same.

In some experiments synthetic UDPG was used as.donor instead of UDPG isolated from yeast. The glycogen obtained was the same with the two samples as judged by molecular weight distribution and acid or alkaline hydrolysis. The reason for carrying out these tests was that UDPG isolated from natural sources may contain other nucleotide sugars and that the introduction of a residue different from glucose might be important in giving glycogen its lability.

\section{DISCUSSION}

A comparison of the glycogen synthesized in vitro from UDPG with that extracted from liver has shown that both exhibit similar molecular weight distribution. The rate and type of breakdown in acid or alkaline solutions is also the same with only minor differences.

The glycogen obtained from UDPG is clearly different from that prepared with glc-1-P. The latter is more stable and its molecular weight is lower when both are synthesized under conditions in which the amount of initial acceptor and of transfer are equal. These facts raise two problems. One is the explanation of the lability which has been discussed in a previous paper (7), and the other is the difference in molecular weight of the glycogens.

It was suggested previously that the lability of native glycogen might be due to the presence of residues different from $\alpha-1,4$ and a-1,6-glucosyl, or to some particular distribution of the monomer units.

It does not seem likely that glycogen prepared in vitro from pure UDPG contains any other residue but glucosyl, However since the enzyme preparation is not pure it cannot be excluded that some unusual kind of linkage could be formed. With reference to the second problem, that is that for the same amount of acceptor and of synthesis, the glycogen obtained from UDPG is much heavier and polydisperse than that prepared with glc-1-P, no clear explanation is as yet available. The difference in the product obtained with the two enzymes may be connected to their different specificity towards the acceptor. 
The transfer of labelled glucose from UD. PG into a phosphorylase limit dextrin was studied by Brown et al. (16). The radioactive product was debranched with a pullulanasetype enzyme and it was found that only $2 \%$ radioactivity was released from the enlarged limit dextrin (9). Therefore, transfer from UDPG occurs on the main chains only. On the other hand Brown et al. (17) studied the action of oligo-glucan-transferase-amylo-1,6glucosidase on phosphorylase limit dextrins enlarged with glc-1-P and concluded that phosphorylase adds randomly to all the nonreducing groups. They also observed that the limit dextrin enlarged with UDPG gave more color with iodine than that grown from glc-1-P. That is as if transfer from UDPG led to the formation of longer branches.

Further evidence showing that glycogen synthetase adds glucose only to some of the exterior chains of glycogen is provided by the work of Kindt and Conrad (18). They found that with an enzyme from Aerobacter aerogenes and adenosine diphosphate glucose as donor, less than half of the non-reducing ends became glucosylated.

\section{REFERENCES}

1. Orrell, S. A., Buening, E., ANd Reissic, M., in Whelan, W. J. and Cameron, M. P. (eds.), "Ciba Foundation Symposium on Control of Glycogen Metabolism," p. 29. Churchill, London, 1964.

2. Barber, A. A., Harris, W. W., AND ANDERSON, N. G., Natl. Cancer Inst. Monograph. 21, 285 (1966).

3. Drochmans, P., J. Ultrastruct. Fes, 6, 141 (1962).

4. Laskov, R., ANd Gross, J., Israel J. Med. Sci. 1, 26 (1965)

5. Mordoh, J., Krisman, C. R., and Leloir, L. F., Arch. Biochem. Biophys. 113, 265 (1966).

6. Morpoh, J., Leloir, L. F., ANd Krisman, C. R., Proc. Natl. Acad. Sci. U. S. 53, 86 (1965).

7. Parobi, A. J., Krisman, C. R., Leloir, L. F., And MornoH, J., Arch. Biochem. Biophys. 121, 769 (1967) .

8. MacDonald, D. L., Methods in Enzymol. 8, 121 (1956).

9. Mofrat, J. G., in Meister, A. (ed.), "Biochemical Preparations," Vol. 8, p. 125. Wiley, New York, 1961.
The results of Biely et al. (19) can be interpreted similarly. They used U.DP-2-deoxyglucose as donor, excess glycogen as acceptor and yeast synthetase. The polysaccharide was then treated with $\beta$-amylase. The products expected from a multichain transfer would be maltose and the analog, containing glucose and 2-deoxyglucose. However, the latter product could not be detected and instead maltose and 2,2'-dideoxymaltose were found, as if repetitive transfer occurred on only some of the outer branches.

If glycogen synthetase adds glucose only to the main chains, the result would be a comblike structure similar to that proposed by Staudinger and Husemann (20) instead of the classical Meyer and Fuld's branched tree (21). The former structure might occur in certain regions of the glycogen molecule so as to produce long chains joining subparticles. These chains would have an increased lability due to the mechanical pull exerted by the subparticles. The difference in molecular weight of the glycogens synthesized from glc-1-P and UDPG seems to be due to a process of aggregation which occurs in the latter case. This problem is being studied.

10. Laskov, R., And Margoliash, E., Bull. Res. Coune. Israel Sect. A11, 351 (1963).

11. Bray, G. A., Anal. Biochem. 1, 279 (1960).

12. Krisman, C. R., Anal. Biochem. 4, 17 (1962).

13. Rothman, L. B., ANd Cabib, E., Biochemistry 6 , 2098 (1967).

14. Krisman, C. R.. Biochim. Biophys. Acta 65, 307 (1962) .

15. Fischer, E. H., Krebs, E. G., ANd Kent, A. D., in Vestling, C. S. (ed.), "Biochemical Preparations," Vol, 6, p. 68. Wiley, New York, 1958.

16. Brown, D. H., Illingworth, B., and Kornfeld, R., Biochemistry. 4, 486 (1965).

17. Brown, D. H., Illingworth, B., And Cori, C. F., Arch. Biochem. Biophys, 116, 479 (1966).

18. Kindt, T. J., ANd Conrad, H. E., Biochemistry 6, 3718 (1967).

19. Biely, P., Farkas, V., And Bauer, S., Biochim. Biophys. Acta 158, 48F (1968).

20. Staudinger, H., and Husemann, E., Liebigs Ann. Chem. 530, 1 (1937).

21. Meyer, K. H., And Fuld, M., Helv. Chim, Acta 24, 375 (1941). 